\title{
Combination of bone marrow mesenchymal stem cells sheet and platelet rich plasma for posterolateral lumbar fusion
}

\author{
Zunpeng Liu' ${ }^{1,2}$, Yue Zhu ${ }^{1}$, Rui Ge ${ }^{3}$, Jiajun Zhu ${ }^{2}$, Xiaoning $\mathrm{He}^{4}$, Xue Yuan ${ }^{5}$ and \\ Xinchun Liu ${ }^{1}$ \\ ${ }^{1}$ Department of Orthopedics, First Affiliated Hospital, China Medical University, Shenyang, China \\ ${ }^{2}$ Department of Orthopedics, Fourth Affiliated Hospital, China Medical University, Shenyang, China \\ ${ }^{3}$ Department of Orthopedics, First Affiliated Hospital, Dalian Medical University, Dalian, China \\ ${ }^{4}$ Department of Stomatology, Fourth Affiliated Hospital, China Medical University, Shenyang, China \\ ${ }^{5}$ Division of Plastic and Reconstructive Surgery, Department of Surgery, Stanford School of Medicine, Stanford, CA, USA \\ Correspondence to: Yue Zhu, email: zhuyuedr_cmulh@126.com
}

Keywords: BMSC, PRP, rabbit posterolateral spinal fusion, osteogenic ability, fusion rate

Received: March 29, $2017 \quad$ Accepted: June 01, $2017 \quad$ Published: July 31, 2017

Copyright: Liv et al. This is an open-access article distributed under the terms of the Creative Commons Attribution License 3.0 (CC BY 3.0), which permits unrestricted use, distribution, and reproduction in any medium, provided the original author and source are credited.

\section{ABSTRACT}

Bone tissue engineering provides a substitute for bone transplantation in spinal fusion. This study examined if combined bone marrow-derived mesenchymal stem cells (BMSCs) sheet with platelet-rich plasma (PRP) could promote bone regeneration in a rabbit posterolateral spinal fusion model. BMSCs was isolated and confirmed by Flow cytometric analysis and immunofluorescence staining. The morphology of BMSCs was examined by Hematoxylin and Eosin staining, scanning and transmission electron microscopy. BMSCs were cultured in induction medium or control medium. The osteogenic ability of BMSCs was investigated by various histochemical staining, immunofluorescence staining and qRT-PCR analysis. The BMSCs/PRP was constructed by encapsulating the PRP block with BMSCs sheet. Twenty-four adult rabbits were randomly divided into four groups based on the implanted biomaterials: BMSCs/ PRP, BMSCs, iliac crest autograft, and control group. Manual palpation and digital radiography analysis showed that the fusion rate was $100 \%, 0,83.3 \%$, and 0 in these 4 groups, respectively. Formation of continuous bone masses in BMSCs/PRP group was confirmed by computed tomography scanning and 3D-reconstruction. These studies demonstrated that BSMCs/PRP significantly accelerated bone regeneration in the rabbit posterolateral spinal fusion model.

\section{INTRODUCTION}

Spinal fusion is one of the most frequently employed surgical techniques for the treatment of various spinal problems including degenerative disc disease with instability, trauma, and deformity. Illiac crest autograft and allogeneic bone are commonly used for lumbar fusion for many years [1]. Following fusion surgery, the most frequent complications are pseudo-arthrosis and donor site diseases $[2,3]$. The occurrence of pseudo-arthrosis is high in iliac crest autograft, and about $50 \%$ of patients reported the donor site problems such as infection, paresthesis, pain, and hematoma using grid instrumentation [4-6].
Therefore, it is important to find suitable substitutes for spinal fusion surgery. Recently, bone tissue engineering has been extensively studied, and three elements are essential for the bone tissue engineering: 1) the osteo conductive scaffold which facilitates neovascularization and supports bone growth; 2) an osteogenic material which provides cells to add in the newly forming bone; and 3) osteo inductive factors which is capable of inducing osteoblastic differentiation from osteoprogenitor stem cells. Bone marrow-derived mesenchymal stem cells (BMSCs) have been shown to be a promising source for tissue regeneration engineering, as they are easily obtained and can be expanded while maintaining their multi 
lineage differentiation potential [7]. In the clinical trials, BMSCs have been applied for the treatment of various diseases including graft-versus-host disease, osteogenesis imperfecta and myocardial infarction [8]. In this regard, BMSCs have shown great potential in the application of bone tissue engineering.

Recently, cell sheet technology has been used in tissue engineering to regenerate damaged soft tissues, including corneal epithelia [9], bladder myocardial cells [10], periodontal ligament cells [11], hepatocytes [12] and so on. Cell sheet technology consists primarily of a "thermo-responsive culture dish" which enables reversible cell adhesion to and detachment from the dish surface by controlling the hydrophobicity of the surface [13]. This allows a non-invasive harvest of high-viability cells in an intact monolayer that includes any deposited extracellular matrix, which is important for the structural and adhesive properties. Similarly, BMSCs sheets can be easily generated and transplanted to the site of large bone defects and may serve as a good candidate for bone tissue regeneration.

Platelet-rich plasma (PRP) is an above-baseline concentration of platelets in plasma derived from centrifugation of autologous blood [14]. Platelets in the circulating plasma are found to contain growth factors that function to enhance soft tissues healing. These growth factors are also important for cell proliferation, differentiation, and neovascularization [15]. PRP has also been shown to contain cell adhesion molecules and chemotactic properties that attract fibroblasts and mesenchymal stem cells to the repair site [16]. Therefore, the healing potential of platelets from PRP may be employed to facilitate bone regeneration.

BMSCs combined with various materials such as hydroxyapatite and $\beta$-tricalcium phosphate, have been found in the application of bone regeneration using cell suspension system $[17,18]$. However, because of the low surface-to-volume ratio of scaffolds, the adhesion rate of BMSCs is very low, which is a big dis-advantage for the cell suspension system. If transplantation of BMSCs without scaffold could enhance bone regeneration at the fusion site, scaffold-free cell-based treatments could be employed, which may represent a better approach to spinal fusion. In this study, we hypothesized that incorporation of an induced BMSC sheet along with PRP would accelerate bone regeneration. We investigated the differentiation of induced BMSCs in vitro and the ability of PRP combined with an induced BMSCs sheet in promoting bone regeneration in a rabbit posterolateral spinal fusion model.

\section{RESULTS}

\section{Isolation of BMSCs}

Freshly isolated bone marrow composed of at least three types of cells: erythrocytes, cells of hemopoietic lineage and mesenchymal cells. In the culture, the erythrocytes never adhere to the substrate, and those hemopoietic cells gradually degenerate or do not survive subculturing in this culture condition. The medium changes removed most of the nonadherent cells during the first four days. After 9-10 days of culture, the predominant cells are morphologically homogeneous spindle-shape cells, most of them are mesenchymal stem cells (Supplementary Figure 1).

\section{Identification of BMSCs with stem cell markers}

To verify that the isolated cells are truly mesenchymal stem cells, we performed flow cytometry to examine the cell surface marks i.e. CD44, CD90, CD31, and CD34 in the isolated cells. As shown in Figure 1A and $1 \mathrm{~B}$, the percentage of CD44 positive cells and CD90 positive cells was $98.1 \%$ and $99 \%$, respectively; while the percentage of $\mathrm{CD} 31$ positive cells and $\mathrm{CD} 34$ positive cells were $3.05 \%$ and $10.7 \%$, respectively (Figure $1 \mathrm{C}$ and 1D). Immunofluorescent staining confirmed that most of the isolated cells are CD44 positive and CD90 positive (Figure $1 \mathrm{E}$ and $1 \mathrm{~F}$ ), indicating that the isolated cells are BMSCs.

\section{Cell viability and cell growth analysis of BMSCs in normal medium and induction medium}

We analyzed the cell viability and cell growth of BMSCs cultured in normal medium and induction medium. The Trypan blue assay showed that there is no significant difference in cell viability between control BMSCs (97.0\%) and induced BMSCs (98.0\%). The cell growth of BMSCs was determined by measuring the cell density, and as shown in Figure 2, there was no significant difference in the cell density of BMSCs grew in control or induction medium.

\section{Morphology analysis of BMSCs cultured in normal or induction medium}

BMSCs grown in the control medium were mostly spindle shape with a few polygonal cells. Most of the BMSCs grown in the induced medium were in triangle or polygonal shape (Figure $3 \mathrm{~A}$ and $3 \mathrm{~B}$ ). Hematoxylin and eosin staining showed that the control BMSCs had basophilic nucleus with clear oval shape, and eosinophilic cytoplasm (Figure 3C); while the induced BMSCs had oval or round nucleus with less eosinophilic cytoplasm (Figure 3D). SEM results showed that the control BMSCs had good cellular morphology with smooth cell surface and spindle shape (Figure 3E); while the induced BMSCs were in triangle or polygonal shapes with clear nucleus, abundant cytoplasm, and the surface of BMSCs showed irregular protrusions, that connect to other cells (Figure 3F). The TEM results showed that the control BMSCs had big nucleus and less cytoplasm with mature organelles 
and abundant ribosomes and mitochondria (Figure 3G); while the induced BMSCs had increased secretory cells with notch nucleus, and there were calcium particles in the cytoplasm and mitochondria, and increased number of homogenous secretory vesicles and rough endoplasmic reticulum. In addition, the induced BMSCs also had abundant mitochondria and Golgi complex (Figure 3H).

\section{Osteogenic ability of induced BMSCs}

We performed immunohistochemistry to examine the expression of collagen I in the induced BMSCs, and the results showed that most of the cells were collagen
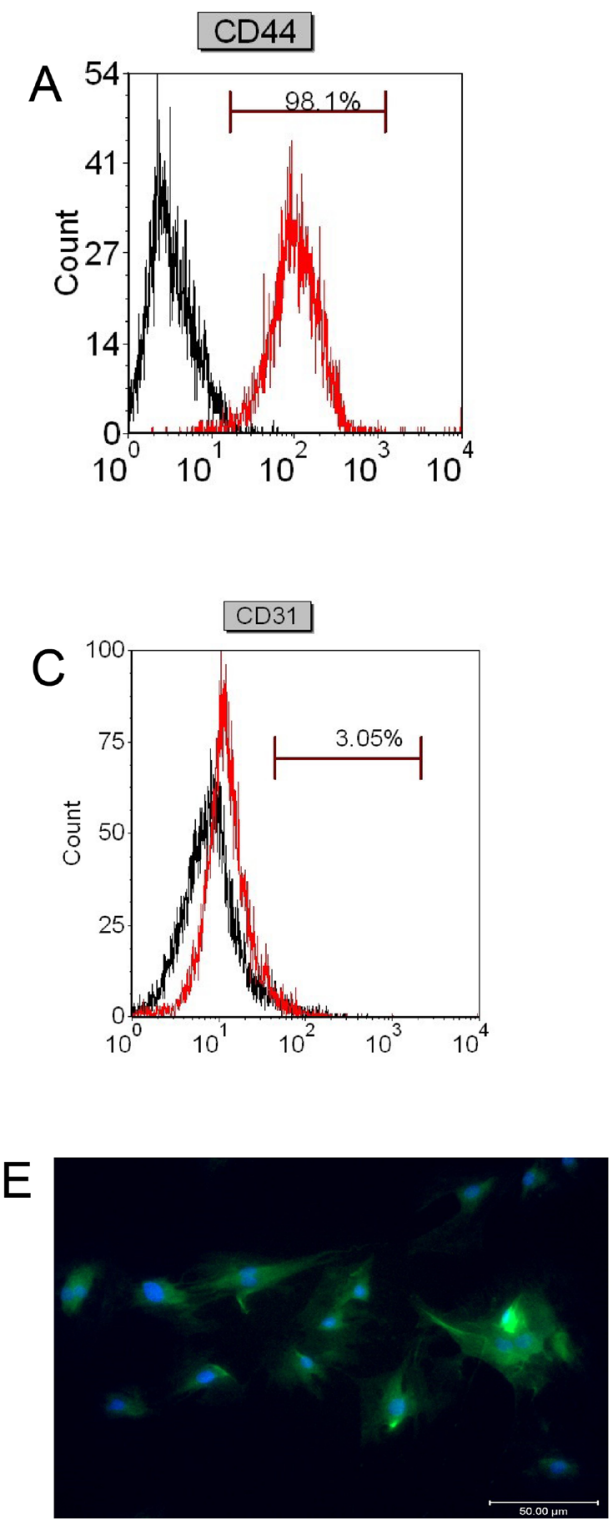

I-positive cells after 5 days of culture (Figure 4A and 4B). In addition, Von Kossa staining of induced BMSCs showed large amounts of black calcium nodules in the cell matrix after 14 days of culture (Figure 4D), and Gomori staining of induced BMSCs showed strong ALP expression after 25 days of culture (Figure 4C). These results demonstrated that these cells have the osteogenic ability.

\section{Morphology and osteogenesis analysis of induced BMSCs sheet}

As shown in Supplementary Figure 2, the induced BMSCs formed cell sheets after 3 weeks of culture, and the
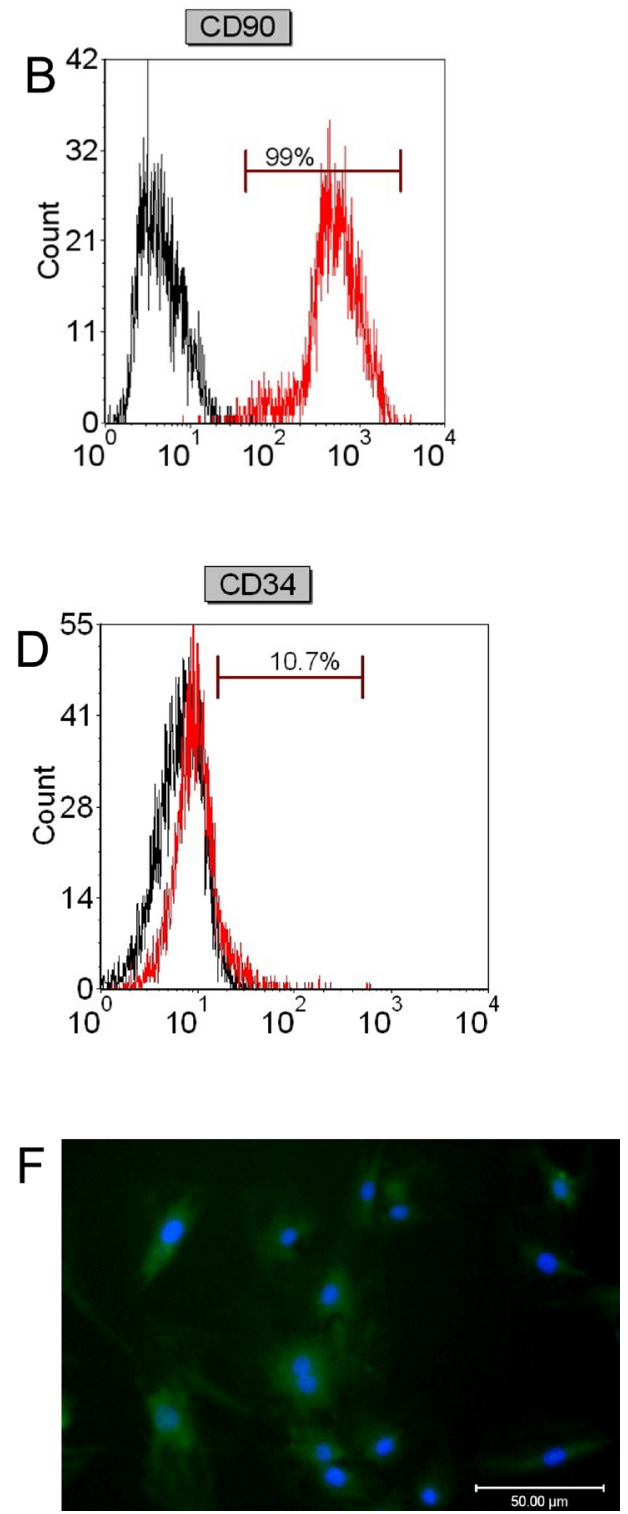

Figure 1: Flow cytometry and immunofluorescence analysis of stem cells markers in isolated BMSCs. Flow cytometry analysis of (A) CD44, (B) CD90, (C) CD31, and (D) CD34 in isolated BMSCs. Immunofluorescent staining of (E) CD44 and (F) CD90 in isolated BMSCs. Scale bar $=50 \mu \mathrm{m}$. 


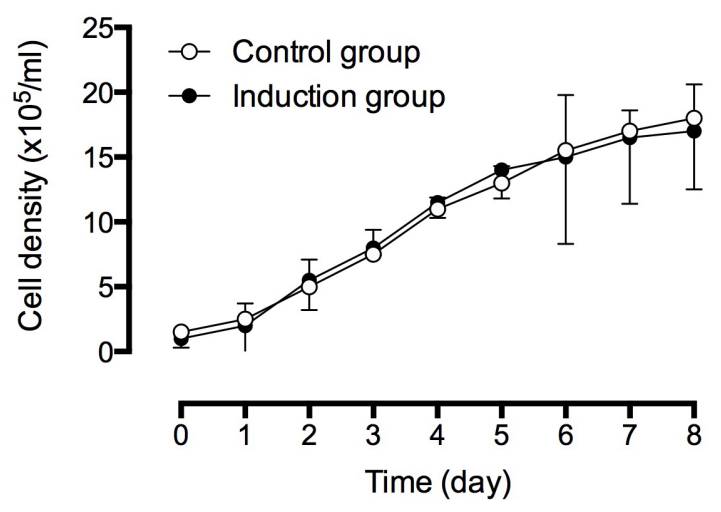

Figure 2: Cell density analysis of BMSCs in control and induced medium. The cell density of cells were continuously monitored for eight days. The experiments were performed in three replicates, and data were presented as mean \pm SD.
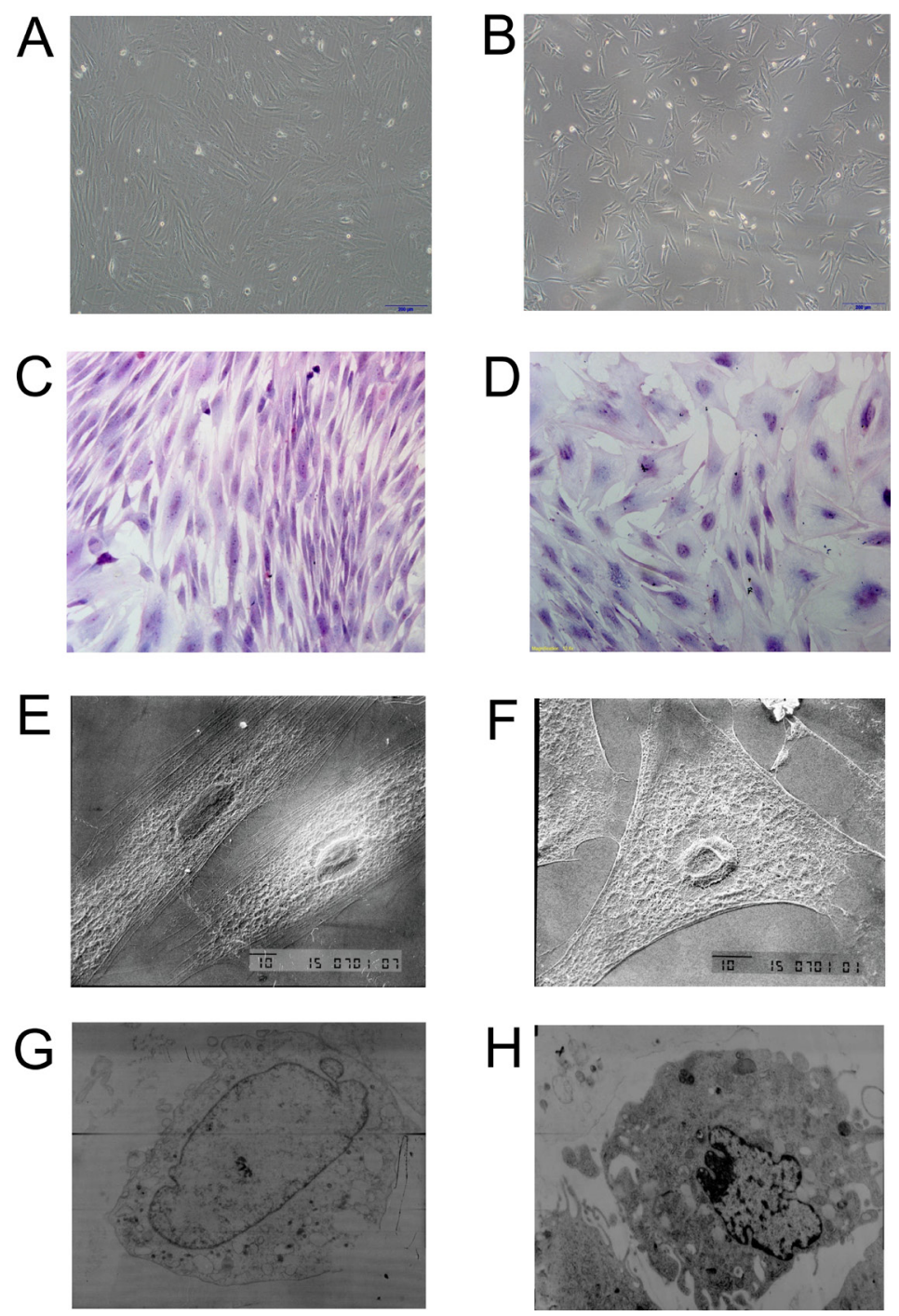

Figure 3: Morphology examination of control BMSCs and induced BMSCs. Inverted microscopy of (A) control BMSCs and (B) induced BMSCs, scale bar $=200 \mu \mathrm{m}$. Hematoxylin and eosin staining of (C) control BMSCs and (D) induced BMSCs, scale bar $=100$ $\mu \mathrm{m}$. SEM images of $\mathbf{( E )}$ control BMSCs and (F) induced BMSCs, scale bar $=10 \mu \mathrm{m}$. TEM images of (G) control BMSCs and (H) induced BMSCs. 
induced BMSCs sheet can be separated from the petri dish by cell scraper. The hematoxylin and eosin staining showed that the induced BMSCs sheet contained a high density of cells, and the cells were evenly distributed and overlapped between layers (Figure 5A and 5B). The SEM results showed that the induced BMSCs sheet formed by BMSCs and the extracellular matrix with tight connection, and there was calcium salt crystallization (Figure 5C and 5D). As shown in Figure 6A, alizarin red staining showed the large amount of calcium nodules formation in the induced BMSCs sheet. Immunofluorescent staining showed the netlike distribution of collagen I in the induced BMSCs sheet (Figure 6B and 6C). Quantitative RT-PCR results showed the expression of OP, ALP and OC mRNAs in the induced BMSCs sheet (Supplementary Figure 3).

\section{Manual and radiographic analysis}

Six weeks after surgery, excised spines from different groups were assessed for fusion after removal of soft tissue by manual palpation. In both control and BMSCs group, no rabbit had spine fusion ( $0 \%$ fusion) (Table 1). In the iliac autograft group, five out of six rabbits had unilateral spine fusion (83.3\% fusion), and in the BMSCs/PRP group, all six rabbits had unilateral spine fusion (100\% fusion) (Table 1). Fisher test showed that BMSCs/PRP group had a significant higher fusion rate than both control group and BMSCs group. Gross
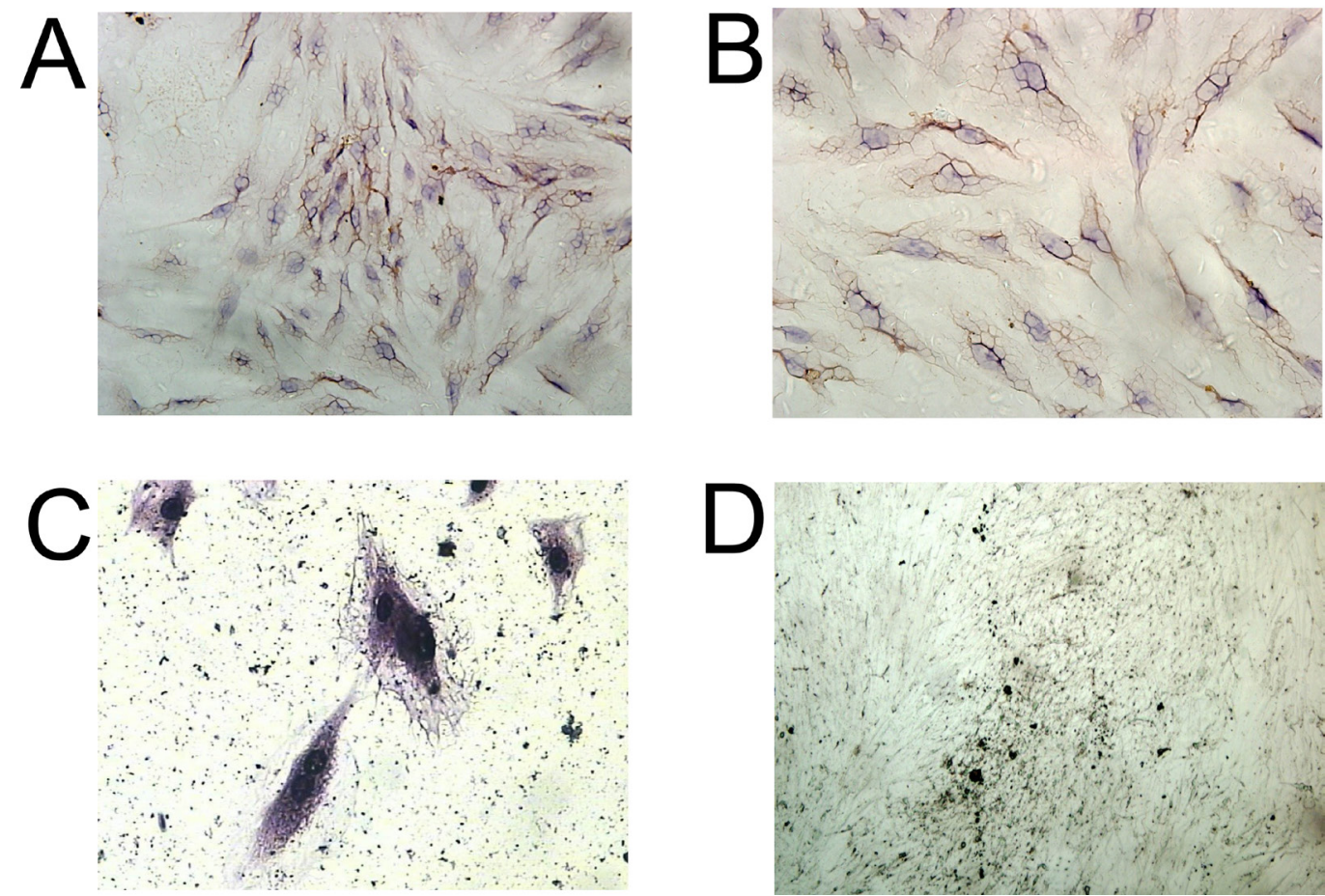

Figure 4: Immunocytochemical analysis of BMSCs. Immunocytochemical staining of Collagen I in (A) control BMSCs, and (B) induced BMSCs, scale bar $=100 \mu \mathrm{m}$. (C) Gomori staining of induced BMSCs, scale bar $=50 \mu \mathrm{m}$. (D) Von kossa staining of induced BMSCs, scale bar $=400 \mu \mathrm{m}$. 

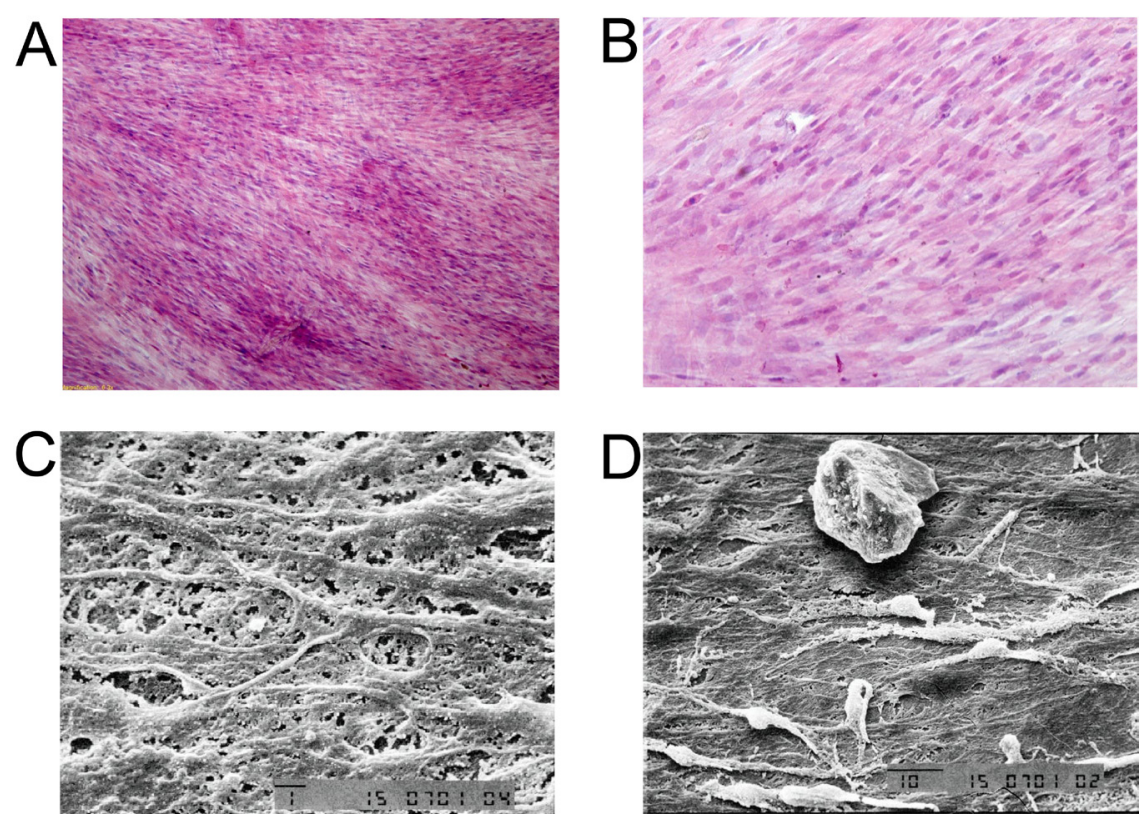

Figure 5: Morphology analysis of BMSCs sheet. Hematoxylin and eosin staining of (A) BMSCs sheet at 100x, scale bar $=400 \mu \mathrm{m}$, and (B) BMSCs sheet at 200x, scale bar $=200 \mu \mathrm{m}$. TEM images of (C) BMSCs sheet at 1500x, scale bar $=1 \mu \mathrm{m}$ and (D) BMSCs sheet at $1000 \mathrm{x}$, scale bar $=10 \mu \mathrm{m}$.
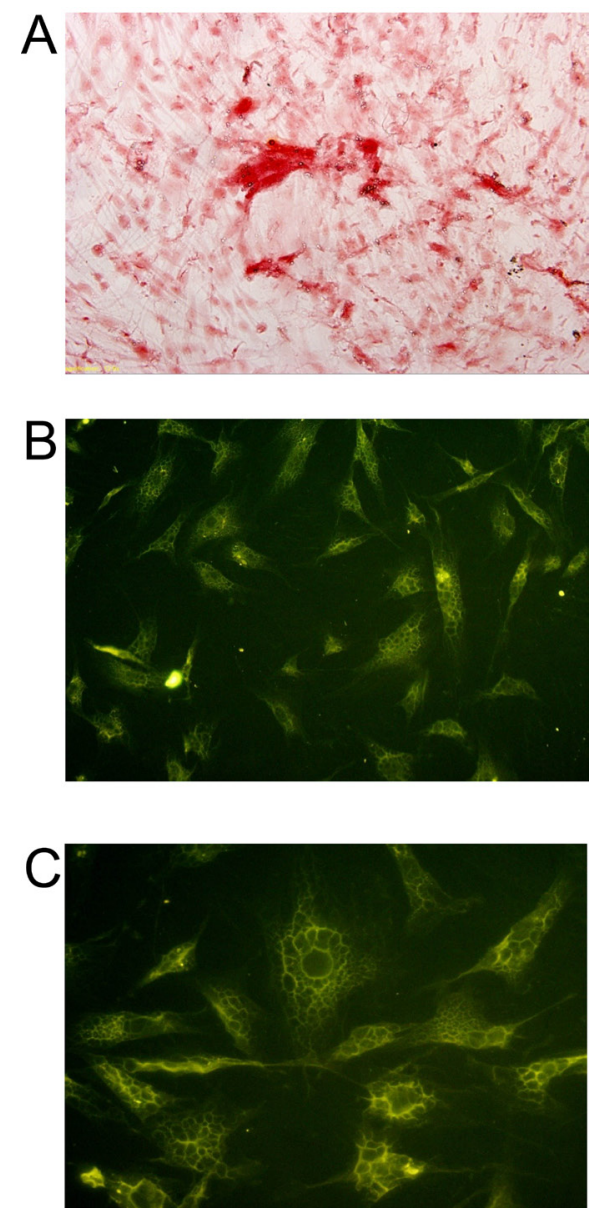

Figure 6: Histochemistry and immunofluorescence analysis of BMSCs sheet. (A) Alizarin Red staining of BMSCs sheet, scale bar $=200 \mu \mathrm{m}$. Immunofluorescent staining of Collagen I (B) in BMSCs sheet at 200x, scale bar $=100 \mu \mathrm{m}$ and (C) in BMSCs sheet at 400x, scale bar $=50 \mu \mathrm{m}$. 
Table 1: Fusion rates (\%) assessed with manual palpation and radiographs

\begin{tabular}{|c|c|c|c|}
\hline Groups & Total (n) & Fusion rate (\%) Manual palpation & Fusion rate (\%) Radiography \\
\hline BMSCs/PRP & 6 & $100 * * \# \#$ & $100 * *$,\#\# \\
\hline BMSCs & 6 & 0 & 0 \\
\hline Iliac autograft & 6 & 83.3 & 83.3 \\
\hline Control & 6 & 0 & 0 \\
\hline
\end{tabular}

Significant differences relative to BMSCs group were indicated as $* * P<0.01$, and significant differences relative to control group were indicated as ${ }^{\#} P<0.01$ (Fisher test).
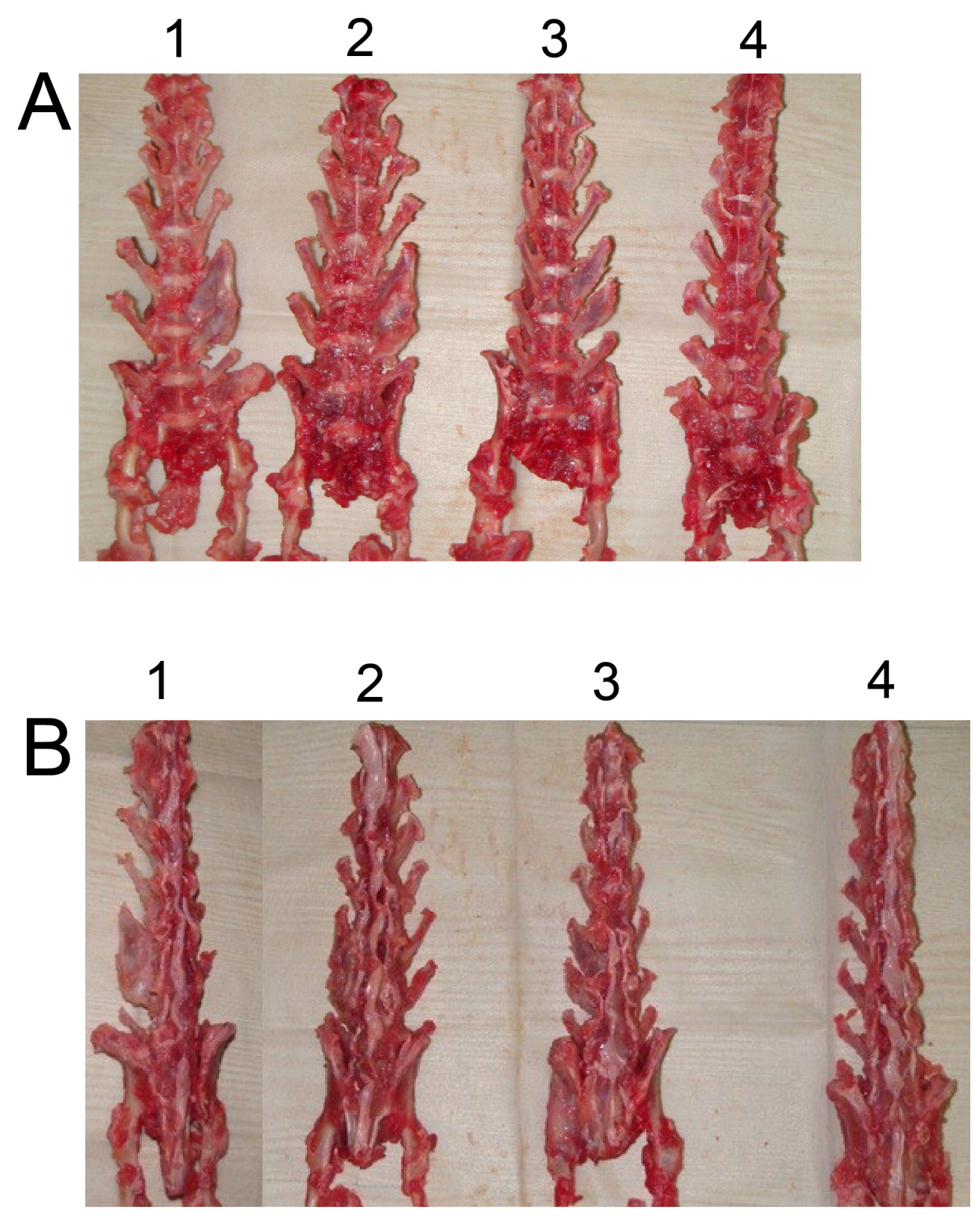

Figure 7: Views of rabbit fusion spines at post-operation 6 weeks. (A) Anterior view of rabbit fusion spines. (B) Posterior view of rabbit fusion spines. 1. BMSCs/PRP group, 2. Iliac crest autograft group, 3. BMSCs group, 4. Control group. 


\section{DISCUSSION}

This study showed that incorporation of the induced BMSCs sheet with PRP greatly promoted bone regeneration in the rabbit spinal fusion model. The induced BMSCs sheet and PRP complex can provide cells and growth factors for the bone regeneration. Importantly, our study revealed that BSMCs/PRP complex had a significant positive effect on the bone regeneration in the rabbit spinal fusion model when compared with BMSCs sheet alone.

In practice, the iliac crest autograft or allogeneic bone was commonly used for the bone transplantation to repair bone defect, bone fracture or spinal fusion. Pseudarthrosis and donor site problems are the most frequent complications following fusion surgery, which largely limited its application [2, 4]. Recently, cell-based tissue engineering provided new strategies for providing suitable candidate biomaterials for bone transplantation.

BMSCs are proved to be one of the most promising seed cells for tissue engineering. Studies have found that BMSCs are multi-potential cells that can be induced to differentiate into several mesodermal cell types including osteoblasts, chondrocytes, adipocytes, tenocytes and myoblasts [21]. In the current study, we isolated the BMSCs from the rabbit bone marrow, and flow cytometry and morphology analysis confirmed the success of BMSCs isolation. Furthermore, the osteogenic ability of the isolated BMSCs was induced
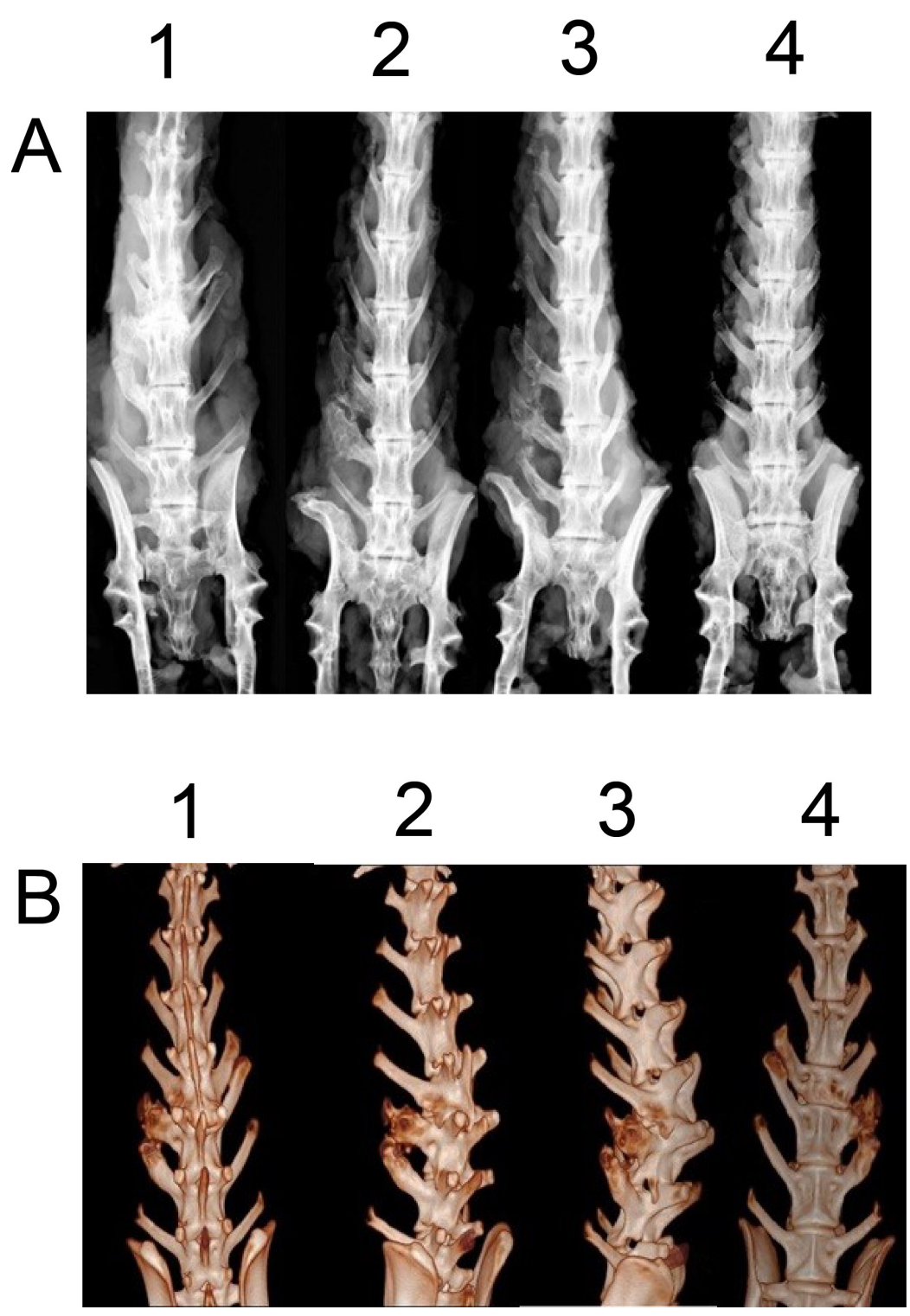

Figure 8: Digital radiography and 3D-CT analysis of rabbit fusion spine at post-operation 6 weeks. (A) Digital radiographic images of rabbit fusion spines. 1. BMSCs/PRP group, 2. Iliac crest autograft group, 3. BMSCs group, 4. Control group. (B) 3D-CT images of lumbar fusion specimens from BMSCs/PRP group. 
by culturing the BMSCs in the induction medium, and confirmed by Von Kossa staining, Gomori staining and the immunohistochemical staining of Collagen I. These results were consistent with previous studies showing that the BMSCs have osteogenic potential under suitable induction medium [22]. In previous studies, BMSCs combined with various materials have been found in the application of bone regeneration by using cell suspension system [23, 24]. However, due to the low surface-to-volume ratio of scaffolds, the adhesion rate of BMSCs is very low. Therefore, in the present study, we adopted a cell transplantation method in which BMSCs are cultured and lifted as a cell sheet, which allows a much larger number of cells to facilitate bone regeneration [25]. The BMSCs sheet has been proven effective in bone regeneration and can effectively preserve cell-to-cell contact and the extracellular matrix [26]. In addition, the layered cell sheet may mimic the in vivo bone deposition of bone matrix where osteoblasts are attached on the mineralized sheet [16]. In this study, The BMSCs sheet can be easily detached from the petridish, and more importantly, the SEM results showed that the extracellular matrix as well as cell-cell interactions remain intact. The immunofluorescence results showed that collagen I was extensively distributed in the BMSCs sheet. In addition, the alizarin red staining was also found to be highly positive. Quantitative RT-PCR analysis further showed the expression of OP, ALP and
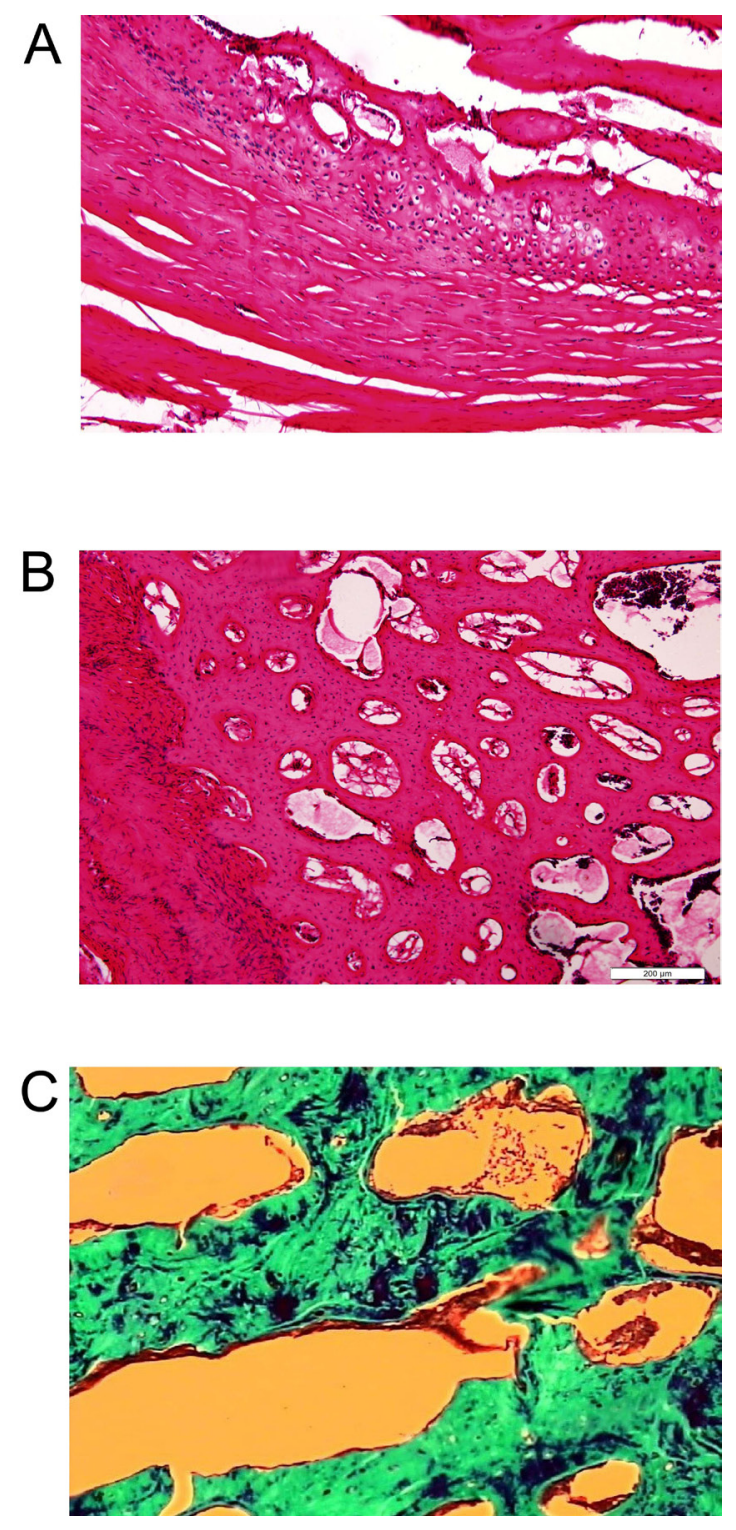

Figure 9: Histology analysis of rabbit spines obtained 6 weeks after surgery. Hematoxylin and eosin staining on sections of the L5-L6 transverse processes of the rabbit spine from fusion sample in BMSCs/PRP group at 10x (A) and 40x (B). (C) Masson's staining on sections of the L5-L6 processes of the rabbit spines from fusion sample in BMSCs/PRP group. 
Table 2: Primers for qRT-PCR

\begin{tabular}{lc}
\hline Genes & Primer sequence \\
\hline Beta-actin & 5'-ATCGTGCGGGACATCAAGGA-3'(F) \\
Osteocalcin & 5'-CAGGAAGGAGGGCTGGAACA-3'(R) \\
& 5'-TCTACCAGTTGCAGCCTGAC-3'(F) \\
Osteopontin & 5'-GTTCCCTTCCTCCTTGATTT-3'(R) \\
& 5'-ACAATATAAGCGCGAGGCCA-3' (F) \\
ALP & 5'-GCTCGATGGCTAGCTTGTCT-3' (R) \\
& 5'-GAGGATGAGAGCAAGGACCC-3' (F) \\
\hline
\end{tabular}

OC mRNAs in the induced BMSCs sheet. These results suggest the osteogenic differentiation of the induced BMSCs sheet.

PRP, which can be easily obtained from blood, is a natural source of growth factors, including platelet derived growth factor, transforming growth factor, insulinlike growth factor, and vascular endothelial growth factor, that can induce osteogenic differentiation of BMSCs [27]. Previous studies showed that BMSCs from PRP-treated mouse had high level of Collagen I and ALP activity [28]. Though the specific growth factors in the PRP was not identified in this study, previous study has shown that the levels of growth factors in PRP samples from goat were higher than those of circulating plasma and PRP can promote the osteogenic differentiation of BMSCs [29, 30]. Owing to the positive effects of BMSCs sheet and PRP on the osteogenesis, we constructed the BMSCs/PRP biomaterials in which the PRP blocks were wrapped by the induced BMSCs sheet and examined the bone regeneration using a rabbit posterolateral spinal fusion model. Manual palpation and radiographic analysis as well as 3D-CT reconstruction of the fused spine showed that BMSCs/ PRP significantly promoted the regenerative response and greatly accelerated bone regeneration compared to other control groups in the rabbit posterolateral spinal fusion model.

One weakness of the present study was that mechanical tests were not performed. In our study, the iliac crest autograft group also had high rate of spinal fusion, and its fusion rate is similar to that in BMSCs/PRP group. However, whether the mechanical property of fused spine in BMSCs/PRP group is similar to or better than that of iliac crest autograft group is unknown. Future study will be performed to examine the mechanical properties of these fused spines.

In summary, our data suggested that the implanted induced BMSCs sheet and PRP play crucial roles in promoting bone regeneration. Our procedure is simple and safe with minimal side effects because both BMSCs sheet and PRP are autologous, nonimmunoreactive and nontoxic. This procedure may be applicable for spinal fusion and could represent a novel alternative to autologous or allogenic bone grafts.

\section{MATERIALS AND METHODS}

\section{Animals}

Adult Japanese white rabbits $(3-5 \mathrm{~kg})$ were purchased from the Animal Department of China Medical University. All animals were housed and treated in strict accordance with the Guidelines on the Care and Use of Laboratory Animals issued by the Chinese Council on Animal Research and the Guidelines of Animal Care. All animal procedures were approved by the China Medical University Laboratory Animal Care.

\section{BMSCs isolation and culture}

Rabbit BMSCs were obtained from the femurs of anesthetized adult Japanese white rabbits. In brief, 10 $\mathrm{ml}$ bone marrow was diluted 1:2 with phosphate buffer solution (PBS) and loaded in a $5 \mathrm{ml}$ Percoll (density, 1.077; PHARMACIA). Cells were harvested from the interface after centrifugation at 2,000 rpm for $20 \mathrm{~min}$ and washed with Dulbecco's modified Eagle's medium (DMEM, Gibco, Grand Island, NY). Cells were resuspended in Low glucose DMEM containing 10\% fetal bovine serum (HYCLONE), $100 \mathrm{U} / \mathrm{ml}$ penicillin, $100 \mathrm{~g} / \mathrm{ml}$ streptomycin, and incubated at $37^{\circ} \mathrm{C}$. When a monolayer was formed in the primary culture, cells were sub-cultured for further experiment.

\section{Induction medium preparation}

The induction medium was prepared as follow: High glucose DMEM, 10\% fetal bovine serum (FBS), $50 \mathrm{mg} / \mathrm{L}$ Vitamin C, $10 \mathrm{mM} \beta$-sodium glycerophosphate, $100 \mathrm{nM}$ dexamethasone, $100 \mathrm{U} / \mathrm{ml}$ penicillin, $100 \mathrm{~g} / \mathrm{ml}$ streptomycin. The induction of BMSCs was achieved 
by sub-culturing the isolated BMSCs in the induction medium.

\section{Preparation of BMSCs sheet}

BMSCs in the third passage was seeded onto the $10 \mathrm{~cm}$ petri-dish with a density of $1 \times 10^{6} / \mathrm{cm}^{2}$, and the BMSCs were cultured with Low glucose DMEM supplemented with $10 \%$ FBS for 2 days, then the BMSCs were cultured under the induction medium for 3 weeks without passage, with the induction medium refreshed every 2 days. After 3 weeks of culture, the semi-transparent thin sheet was observed at the bottom of the petri-dish (Supplementary Figure 2B). The BMSCs sheet was separated from the petri-dish by a cell scraper (Supplementary Figure 2C).

\section{Flow cytometric analysis of BMSCs using CD44, CD90, CD31 and CD34 biomarkers}

For the flow cytometric analysis, fluorescein isothiocyanate (FITC) conjugated antibodies against CD44, CD90, CD31, and CD34 (Dako corporation, Glostrup, Denmark) were used. Isolated cells cultured in the induction medium from the third passage were used. First, cells attached to the bottom of the flask were detached by Trypsin/EDTA treatment. Then, the appropriate concentration of the above-mentioned antibodies (1:10 dilution) was added, and incubated at room temperature for 20 min at dark. After that, the cells were washed with PBS and analyzed by flow cytometry using the FITC method and a FACS Calibur (Becton Dickinson).

\section{Immunofluorescent staining for BMSCs cells}

The BMSCs cells were treated with $0.3 \%$ Triton, and were blocked at room temperature for $90 \mathrm{~min}$ before incubation with FITC-conjugated antibodies against CD44 and $\mathrm{CD} 90$ at $4^{\circ} \mathrm{C}$ for $30 \mathrm{~min}$. Then the cells were stained with 4',6-diamidino-2-phenylindole at room temperature for $10 \mathrm{~min}$. The fluorescent signals were examined under a fluorescent microscope.

\section{Cell viability and cell growth assays}

Isolated cells from the third passage were used for the assays. Cell viability was examined using the tryphan blue assay. $1 \times 10^{5} / \mathrm{ml}$ cells were seeded on the six-well plate and cultured with either normal medium or induction medium. After culture for three days, $20 \mu \mathrm{l}$ of cell suspension was mixed with $80 \mu$ lysing solution (Beckman Coutler, Marseille Cedex, France) for $5 \mathrm{~min}$, and then mixed with $100 \mu$ tryphan blue solution $(0.4 \%$ $\mathrm{w} / \mathrm{v}, \mathrm{GIBCO})$. The samples were loaded into a Neubauer hemocytometer, and the cells in each $1 \mathrm{~mm}^{2}$ area were counted under a light microscope. For the cell growth assay, $1 \times 10^{5} / \mathrm{ml}$ cells were seeded on the 48 -well plate and were cultured with either Low glucose DMEM medium or induction medium. The cell number was counted under a light microscope for consecutive 8 days.

\section{Hematoxylin and eosin staining}

The Hematoxylin and eosin staining of isolated BMSCs and BMSCs sheets were performed according to previous protocol [19].

\section{Scanning electron microscopy (SEM)}

The specimens were submerged in fixative $(2.5 \%$ glutaraldehyde in phosphate buffer at $\mathrm{pH}=7.3$ ) overnight. Specimens were dehydrated using graded alcohol and sputter coated with gold. SEM imaging of specimens was conducted at various magnifications.

\section{Transmission electron microscopy (TEM)}

The specimens were fixed in $2.5 \%$ glutaraldehyde in $0.1 \mathrm{M}$ phosphate buffer for 2 hours. The samples were washed with $0.1 \mathrm{M}$ phosphate buffer, post-fixed in $1 \%$ OsO4 buffered with $0.1 \mathrm{M}$ phosphate buffer for 2 hours, dehydrated in a graded series of ethanol and embedded in Epon 812. Specimens were collected on copper grids, double-stained with uranyl acetate and lead citrate, and then examined by transmission electron microscopy.

\section{Immunocytochemical staining and immunofluorescent staining of collagen I}

The immnuocytochemical staining was performed to examine the distribution of collagen $\mathrm{I}$ in the induced BMSCs. Briefly, the BMSCs were fixed in formalin for 20 min and incubated with $1 \% \mathrm{H}_{2} \mathrm{O}_{2}$ for $10 \mathrm{~min}$. After that, the samples were blocked with goat serum for $30 \mathrm{~min}$ at room temperature, followed by incubation with primary antibody (anti-collagen I mouse monoclonal antibody) at $4^{\circ} \mathrm{C}$ overnight. After primary antibody incubation, cells were further incubated with the HRP-conjugated secondary antibody, and the immnuocytochemical staining was visualized by incubation with 3,3'-Diaminobenzidine. Images of the immunocytochemical staining were examined under a light microscope.

For immunofluorescence staining, BMSCs sheet were fixed with $4 \%$ paraformaldehyde for $10 \mathrm{~min}$, and blocked for $30 \mathrm{~min}$ in 5\% normal goat serum. After that, samples were incubated with primary antibody(anticollagen I mouse monoclonal antibody) for 12 hours. Then, they were incubated with secondary antibody (FITC conjugated secondary antibody, Sigma, F0382) for 2 hours 
and the nucleus was subsequently counterstained by DAPI. The slides were mounted with mounting medium, and the fluorescent signals were examined under a fluorescent microscope.

\section{von Kossa staining}

The presence of mineralized deposits in induced BMSCs cultures was demonstrated with von Kossa staining. The induced BMSCs were rinsed twice with Tyrode's salt solution, fixed with $1 \%$ glutaraldehyde (v /v) for $15 \mathrm{~min}$, and rinsed three times with distilled water. One $\mathrm{ml}$ of $2 \%(\mathrm{w} / \mathrm{v})$ silver nitrate (Sigma Chemical) was added per dish following $10 \mathrm{~min}$ incubation in a dark environment. Cultures were then rinsed $3 \mathrm{X}$ times with distilled water and exposed to bright light for $15 \mathrm{~min}$. Cultures were rinsed and then dehydrated with 100\% ethanol.

\section{Gomori staining}

Induced BMSCs were fixed in cold 10\% neutral buffer formalin for 1 hour at $4^{\circ} \mathrm{C}$. Then, the cell layers were washed with deionized water and allowed to air dry. The fixed cells were incubated with buffer containing 0.1 $\mathrm{mg} / \mathrm{ml}$ naphthol AS-MX phosphate disodium salt (Sigma) and $0.6 \mathrm{mg} / \mathrm{ml}$ Fast Red TR salt (Sigma). After 1 hour at $37^{\circ} \mathrm{C}$, the cell layers were washed with deionized water and observed both grossly and with the light microscope.

\section{Alizarin red histochemical staining}

The BMSCs sheet was fixed in cold $70 \%$ ethanol for 1 hour at $4^{\circ} \mathrm{C}$. Then, the cell layers were washed with deionized water and allowed to air dry. The fixed cells were stained with 2\% Alizarin red pH 7.2 (Sigma). After 1 hour at $37^{\circ} \mathrm{C}$, the cell layers were washed with deionized water and observed both grossly and with the light microscope.

\section{Quantitative RT-PCR}

Total RNA was extracted from the cells using TirZol reagent. The extracted RNA was then reversetranscribed into single stranded cDNA, using MMLV Reverse Transcriptase and Oligo(dT) primers according to the manufacturer's instructions (Promega). For gene expression analysis, real-time PCR was performed using SYBR-Green-based protocols in an ABI Step One system. The expression levels of target genes in each sample were calculated after being normalized to the $\mathrm{Ct}$ value of the beta-actin housekeeping gene. The primers for respective genes include osteopontin(OP), alkaline phosphatase (ALP) and osteocalcin (OC)were shown in Table 2.

\section{Preparation of PRP and implanted biomaterials}

Autologous PRP was prepared as described previously [20]. Briefly, $10 \mathrm{~mL}$ of whole blood was drawn from the marginal auricular vein using an 18-gauge catheter. The blood was injected into a sterile centrifuge tube containing $1.5 \mathrm{~mL}$ of sodium citrate. The mixture was centrifuged at $1200 \times \mathrm{g}$ for $10 \mathrm{~min}$ to separate the plasma from the red blood cells. The plasma was centrifuged again at $2500 \times \mathrm{g}$ at $4^{\circ} \mathrm{C}$ for $20 \mathrm{~min}$, and the precipitated platelets $(1 \mathrm{~mL})$ were collected. The prepared autologous PRP was settled in a customized mold (Supplementary Figure 4A), and the PRP was placed on the BMSCs sheet (Supplementary Figure 4B). Then the implanted biomaterials were prepared by wrapping the PRP with the detached BMSCs sheet.

\section{Surgical procedures for biomaterial implantation}

Rabbits ( $\mathrm{n}=24)$ were subcutaneously injected with buprenoprhine $30 \mathrm{~min}$ before surgery, and the surgical site was shaved and prepped with betadine and 70\% ethanol. The rabbits were anesthetized with intravenous injection of $25 \mathrm{mg} / \mathrm{kg}$ sodium pentobarbital. Aseptic technique was used for all surgical procedures. The L6 vertebral body was identified using the iliac crest as a landmark. A 6-cm longitudinal midline incision was made through the skin and subcutaneous tissue over L5-L6 down to the lumbodorsal fascia. A 3-cm longitudinal paramedial incision was then made in the paraspinal muscles bilaterally to expose the transverse processes of L5 and L6, which were decorticated with a high-speed burr. The surgical site was then irrigated with sterile saline, and 2 $\mathrm{cm}$ X $1.5 \mathrm{~cm} \mathrm{X} 0.5 \mathrm{~cm}$ pieces of biomaterial (BMSCs/ PRP group, iliac crest autograft group, BMSCs group, control group) were placed unilaterally (Supplementary Figure 4C and 4D), with each implant spanning the transverse processes. All the implanted biomaterials were randomly assigned to each animal. The implants were then covered with the overlying paraspinal muscles, and the lumbodorsal fascia and skin were closed with 4-0 Prolene sutures (Ethicon, Inc., Somerville, NJ, USA). Animals were allowed to ambulate, eat, and drink ad libitum immediately after surgery.

\section{Manual assessment of fusion}

Six weeks post surgery, animals were euthanized and the spines were surgically removed and blindly evaluated by three independent observers for motion between levels after removal of soft tissue. Nonunion was recorded if motion was observed between the facets or transverse processes on surgical side. Complete fusion was recorded if no motion was observed. Spines were scored 
as either fused or not fused. Unanimous agreement was required to consider a spine completely fused.

\section{Radiographic assessment of fusion}

Radiographs of the lumbar spine were taken for each animal at 6 weeks after surgery using a Faxitron LX60 cabinet radiography system for posteroanterior images. Radiographs were blindly evaluated by three independent observers. Spines were scored as either fused or not fused. Unanimous agreement was required to consider a spine completely fused.

\section{Computed tomography (CT) and three-dimensional (3D) reconstruction}

The spine from BMSCs/PRP group was scanned by CT using LightSpeed 16 (GE, Milwaukee, WI), and the data were collected at $80 \mathrm{kV}$ and $300 \mathrm{~mA}$, and reconstructed using the cone-beam algorithm.

\section{Histological analysis}

Six weeks post implantation, the spines were dissected, and the specimens were fixed in $40 \%$ ethanol, decalcified using standard $10 \%$ decalcifying solution $\mathrm{HCl}$ (Cal-Ex; Fischer Scientific, Fairlawn, NJ), washed with running tap water, and then transferred to $75 \%$ ethanol. Serial coronal sections between the L5-L6 transverse processes were cut carefully at the left sides. The specimens were embedded in wax for sectioning. Coronal sections $(5 \mu \mathrm{m})$ were cut from the paraffin blocks using a microtome (LS-113; DAIWA-KOKI, Saitama, Japan). The sections were then stained with hematoxylin and eosin for basic morphology or Masson (Masson kit, Zhuchun, Co.Ltd., Shanghai) for collagen morphology.

\section{Statistical analysis}

The statistical analyses were performed using the SPSS software package (Version 12.0). The differences among groups were analyzed by Chi-square test. All data are expressed as mean $\pm \mathrm{SD}$. Differences were considered significant when $\mathrm{P}<0.05$.

\section{CONFLICTS OF INTEREST} interest.

The authors declare that they have no competing

\section{REFERENCES}

1. Herkowitz HN, Kurz LT. Degenerative lumbar spondylolisthesis with spinal stenosis. A prospective study comparing decompression with decompression and intertransverse process arthrodesis. J Bone Joint Surg Am. 1991; 73:802-08.
2. Thomsen K, Christensen FB, Eiskjaer SP, Hansen ES, Fruensgaard S, Bünger CE. 1997 Volvo Award winner in clinical studies. The effect of pedicle screw instrumentation on functional outcome and fusion rates in posterolateral lumbar spinal fusion: a prospective, randomized clinical study. Spine. 1997; 22:2813-22.

3. Glassman SD, Dimar JR 3rd, Burkus K, Hardacker JW, Pryor PW, Boden SD, Carreon LY. The efficacy of rhBMP-2 for posterolateral lumbar fusion in smokers. Spine. 2007; 32:1693-98.

4. Summers BN, Eisenstein SM. Donor site pain from the ilium. A complication of lumbar spine fusion. J Bone Joint Surg Br. 1989; 71:677-80.

5. Banwart JC, Asher MA, Hassanein RS. Iliac crest bone graft harvest donor site morbidity. A statistical evaluation. Spine. 1995; 20:1055-60.

6. Silber JS, Anderson DG, Daffner SD, Brislin BT, Leland JM, Hilibrand AS, Vaccaro AR, Albert TJ. Donor site morbidity after anterior iliac crest bone harvest for singlelevel anterior cervical discectomy and fusion. Spine. 2003; 28:134-39.

7. $\mathrm{Wu} \mathrm{W}$, Le AV, Mendez JJ, Chang J, Niklason LE, Steinbacher DM. Osteogenic performance of donormatched human adipose and bone marrow mesenchymal cells under dynamic culture. Tissue Eng Part A. 2015; 21:1621-32.

8. de Girolamo L, Lucarelli E, Alessandri G, Avanzini MA, Bernardo ME, Biagi E, Brini AT, D’Amico G, Fagioli F, Ferrero I, Locatelli F, Maccario R, Marazzi M, et al. Mesenchymal stem/stromal cells: a new "cells as drugs" paradigm. Efficacy and critical aspects in cell therapy. Curr Pharm Des. 2013; 19:2459-73.

9. Nishida $K$, Yamato $M$, Hayashida $Y$, Watanabe $K$, Maeda N, Watanabe H, Yamamoto K, Nagai S, Kikuchi A, Tano Y, Okano T. Functional bioengineered corneal epithelial sheet grafts from corneal stem cells expanded ex vivo on a temperature-responsive cell culture surface. Transplantation. 2004; 77:379-85.

10. Shimizu T, Sekine H, Isoi Y, Yamato M, Kikuchi A, Okano T. Long-term survival and growth of pulsatile myocardial tissue grafts engineered by the layering of cardiomyocyte sheets. Tissue Eng. 2006; 12:499-507.

11. Hasegawa M, Yamato M, Kikuchi A, Okano T, Ishikawa I. Human periodontal ligament cell sheets can regenerate periodontal ligament tissue in an athymic rat model. Tissue Eng. 2005; 11:469-78.

12. Harimoto M, Yamato M, Hirose M, Takahashi C, Isoi Y, Kikuchi A, Okano T. Novel approach for achieving doublelayered cell sheets co-culture: overlaying endothelial cell sheets onto monolayer hepatocytes utilizing temperatureresponsive culture dishes. J Biomed Mater Res. 2002; 62:464-70.

13. Takezawa T, Mori Y, Yoshizato K. Cell culture on a thermoresponsive polymer surface. Biotechnology (N Y). 1990; 8:854-56. 
14. Civinini R, Macera A, Nistri L, Redl B, Innocenti M. The use of autologous blood-derived growth factors in bone regeneration. Clin Cases Miner Bone Metab. 2011; 8:25-31.

15. Tsai WC, Yu TY, Lin LP, Lin MS, Wu YC, Liao CH, Pang JS. Platelet rich plasma releasate promotes proliferation of skeletal muscle cells in association with upregulation of PCNA, cyclins and cyclin dependent kinases. Platelets. 2016; 28: 491-497.

16. Teng C, Zhou C, Xu D, Bi F. Combination of platelet-rich plasma and bone marrow mesenchymal stem cells enhances tendon-bone healing in a rabbit model of anterior cruciate ligament reconstruction. J Orthop Surg. 2016; 11:96.

17. Vila M, García A, Girotti A, Alonso M, Rodríguez-Cabello JC, González-Vázquez A, Planell JA, Engel E, Buján J, García-Honduvilla N, Vallet-Regí M. 3D silicon doped hydroxyapatite scaffolds decorated with Elastin-like Recombinamers for bone regenerative medicine. Acta Biomater. 2016; 45:349-56.

18. Gao P, Zhang H, Liu Y, Fan B, Li X, Xiao X, Lan P, Li M, Geng L, Liu D, Yuan Y, Lian Q, Lu J, et al. Beta-tricalcium phosphate granules improve osteogenesis in vitro and establish innovative osteo-regenerators for bone tissue engineering in vivo. Sci Rep. 2016; 6:23367.

19. Syed-Picard FN, Shah GA, Costello BJ, Sfeir C. Regeneration of periosteum by human bone marrow stromal cell sheets. J Oral Maxillofac Surg. 2014; 72:1078-83.

20. Wu W, Chen F, Liu Y, Ma Q, Mao T. Autologous injectable tissue-engineered cartilage by using platelet-rich plasma: experimental study in a rabbit model. J Oral Maxillofac Surg. 2007; 65:1951-57.

21. Xiao Y, Mareddy S, Crawford R. Clonal characterization of bone marrow derived stem cells and their application for bone regeneration. Int J Oral Sci. 2010; 2:127-35.

22. Kagami H, Agata H, Inoue M, Asahina I, Tojo A, Yamashita $\mathrm{N}$, Imai $\mathrm{K}$. The use of bone marrow stromal cells (bone marrow-derived multipotent mesenchymal stromal cells) for alveolar bone tissue engineering: basic science to clinical translation. Tissue Eng Part B Rev. 2014; 20:229-32.
23. Dumas A, Moreau MF, Ghérardi RK, Baslé MF, Chappard D. Bone grafts cultured with bone marrow stromal cells for the repair of critical bone defects: an experimental study in mice. J Biomed Mater Res A. 2009; 90:1218-29.

24. Giannoni P, Mastrogiacomo M, Alini M, Pearce SG, Corsi A, Santolini F, Muraglia A, Bianco P, Cancedda R. Regeneration of large bone defects in sheep using bone marrow stromal cells. J Tissue Eng Regen Med. 2008; 2:253-62.

25. Ouyang HW, Toh SL, Goh J, Tay TE, Moe K. Assembly of bone marrow stromal cell sheets with knitted poly (L-lactide) scaffold for engineering ligament analogs. J Biomed Mater Res B Appl Biomater. 2005; 75:264-71.

26. Nakamura A, Akahane M, Shigematsu H, Tadokoro M, Morita Y, Ohgushi H, Dohi Y, Imamura T, Tanaka Y. Cell sheet transplantation of cultured mesenchymal stem cells enhances bone formation in a rat nonunion model. Bone. 2010; 46:418-24.

27. Lindner T, Kanakaris NK, Marx B, Cockbain A, Kontakis G, Giannoudis PV. Fractures of the hip and osteoporosis: the role of bone substitutes. J Bone Joint Surg Br. 2009; 91:294-303.

28. Duan J, Kuang W, Tan J, Li H, Zhang Y, Hirotaka K, Tadashi K. Differential effects of platelet rich plasma and washed platelets on the proliferation of mouse MSC cells. Mol Biol Rep. 2011; 38:2485-90.

29. Bi L, Cheng W, Fan H, Pei G. Reconstruction of goat tibial defects using an injectable tricalcium phosphate/chitosan in combination with autologous platelet-rich plasma. Biomaterials. 2010; 31:3201-11.

30. Zhou Y, Ni Y, Liu Y, Zeng B, Xu Y, Ge W. The role of simvastatin in the osteogenesis of injectable tissueengineered bone based on human adipose-derived stromal cells and platelet-rich plasma. Biomaterials. 2010; 31:5325-35. 